\title{
Green Vehicle Routing Optimization Based on Carbon Emission and Multiobjective Hybrid Quantum Immune Algorithm
}

\author{
Xiao-Hong Liu, ${ }^{1}$ Mi-Yuan Shan $\left(\mathbb{D},{ }^{1}\right.$ Ren-Long Zhang, ${ }^{2}$ and Li-Hong Zhang ${ }^{3}$ \\ ${ }^{1}$ College of Business Administration, Hunan University, No. 11 Lushan South Road, Changsha 410082, China \\ ${ }^{2}$ Business School, Hunan University of Science and Technology, Taoyuan Road, Xiangtan 411201, China \\ ${ }^{3}$ Liverpool Business School, Liverpool John Moores University, Redmonds Building, Brownlow Hill, Liverpool L3 5UX, UK \\ Correspondence should be addressed to Mi-Yuan Shan; shanmiyuangj@163.com
}

Received 16 November 2017; Accepted 28 February 2018; Published 11 April 2018

Academic Editor: Salvatore Strano

Copyright (C) 2018 Xiao-Hong Liu et al. This is an open access article distributed under the Creative Commons Attribution License, which permits unrestricted use, distribution, and reproduction in any medium, provided the original work is properly cited.

\begin{abstract}
Green Vehicle Routing Optimization Problem (GVROP) is currently a scientific research problem that takes into account the environmental impact and resource efficiency. Therefore, the optimal allocation of resources and the carbon emission in GVROP are becoming more and more important. In order to improve the delivery efficiency and reduce the cost of distribution requirements through intelligent optimization method, a novel multiobjective hybrid quantum immune algorithm based on cloud model (CHQIA) is put forward. Simultaneously, the computational results have proved that the C-HQIA is an efficient algorithm for the GVROP. We also found that the parameter optimization of the C-HQIA is related to the types of artificial intelligence algorithms. Consequently, the GVROP and the C-HQIA have important theoretical and practical significance.
\end{abstract}

\section{Introduction}

With the increasing global average temperature and global energy tension, climate change has become a global concern. Energy saving and emission reduction have become a common responsibility of the international community. The main source of carbon dioxide is burning fossil fuels. Changing the structure of energy consumption and reducing the proportion of fossil fuels in energy consumption can improve the efficiency of energy utilization. Green logistics refers to the suppression of logistics in the process of logistics which is harmful to the environment. At the same time, it is very important to achieve the purification of the logistics environment and to make full use of logistics resources. The main objective is also to reduce environmental pollution and reduce energy consumption. Consequently, green logistics is a sustainable development of the concept of logistics.

We all knew that transportation is the main way of oil consumption and air pollution emissions. The total energy consumption can account for $25 \%$ to $30 \%$. From the view of energy consumption structure, the energy consumption is mainly focused on fuel consumption in the transportation field. It mainly consumes gasoline, kerosene, diesel, and other kinds of refined oil in the fuel consumption. Fuel oil consumption is relatively small and crude oil is the least consumed material. With the rapid growth of traffic, the energy consumption will continue to grow in the transportation sector. Its growth rate is much higher than the growth rate of energy consumption in the whole society. Its energy consumption accounts for the proportion of energy consumption in society as a whole which continues to increase. Therefore, the green path optimization problem is becoming more and more important.

The vehicle routing problem (VRP) is a complex and high-level set of routing problems. One of its important variants is the dynamic vehicle routing problem (DVRP) in which not all customers are known in advance. The DVRP is a dynamic optimization problem (DOP) that has become a challenging research topic. Considering dynamic demand, time window, and customer satisfaction, the dynamic multiobjective dynamic vehicle routing model is constructed and the model can describe the problem of green logistics distribution better (AbdAllah et al., 2017) [1]. We also know that transportation is one of the most important functional 
elements in logistics. Therefore, rationalization of logistics largely depends on rationalization of transportation, through green transportation route optimization and guide enterprises to implement green supply chain management. At the same time, this paper focuses on a core issue in energy saving emission reduction and attempts to achieve improvement of environmental quality.

\section{Vehicle Routing Optimization}

The VRP is considered to produce routes and schedules for a fleet of delivery vehicles that minimize the fuel emissions in a road network where speeds depend on time. In the model, the route for each vehicle must be determined and also the speeds of the vehicles along each path are treated as decision variables. According to the decomposition condition of vehicle departure, the transformation function of speed travel time and speed carbon emission is given in general. The travel time model and the carbon emission model are constructed according to the changing attributes of speed (Qian and Eglese, 2016) [2]. The VRP and firefly algorithms are studied and a multiobjective mathematical model is established to minimize the number of vehicles. The discrete firefly algorithm based on variable neighborhood search algorithm is also proposed (Marinaki and Marinakis, 2016) [3]. The routing problem and the integrated inventory transportation problem are studied from two aspects: model and algorithm. Different classification methods and different modeling methods of vehicle routing problem are introduced (Lefever et al., 2016) [4].

The capacitated vehicle routing problem (CVRP) has been proved to be NP complete problem. According to the characteristics of combinatorial optimization problems, the discrete hybrid weed optimization algorithm is proposed to solve the traveling salesman problem. The single point sequential crossover method and mutation method are introduced to prevent the premature convergence of the algorithm. The computer simulation results show that the discrete hybrid weed optimization algorithm has better performance (Zhao et al., 2016) [5]. The general vehicle routing problem with time windows based on the improved tabu search algorithm is solved for the vehicle routing problem. In the improved algorithm, the better solution is selected as the initial solution and the dynamic tabu table is constructed to change the size and structure of tabu table with the search process. Simulation results show the feasibility, effectiveness, and superiority of the proposed algorithm (Sicilia et al., 2016) [6]. The vehicle routing problem with hard time windows and stochastic service times (VRPTW-ST) is introduced in the form of a chance-constrained model and mainly differs from other vehicle routing problems with stochastic service or travel times considered by the presence of hard time windows (Errico et al., 2016) [7].

To measure the efficiency of the problem, an improved particle swarm optimization (IPSO) algorithm is proposed and it is compared with an original PSO algorithm in terms of accuracy. The improved algorithm is used to solve the vehicle routing problem with time window and weight limit which is closer to the logistics management problem in real life
(Norouzi et al., 2015) [8]. An adaptive nonlinearly decreasing modulation model is used to keep a well balance between the exploration and exploitation. The proposed algorithm is superior and confirms its potential to solve vehicle routing problem with time windows (VRPTW) (Tan et al., 2015) [9]. The proposed multiobjective optimization model tackles the conflicting objectives of the emission reduction while holding-off the economic cost uplift leading to a set of Pareto optimal solutions. The vehicle routing problem with time window constraints with carbon emissions is an extension of the classical vehicle routing problem. However, the optimization objectives and constraints are more complex (Jabir et al., 2015) [10]. Time dependent vehicle routing problem (TDVRP) is the study of vehicle routing optimization in the road network environment with changing departure time. Therefore, it is more difficult to solve the TDVRP with timevarying traffic characteristics. The initial feasible solution of ant colony algorithm is generated by the nearest neighbor method for TDVRP based on the minimum cost. The quality of the feasible solution is improved by local search operation (Ding et al., 2012) [11].

Considering the uncertainty of vehicle travel time and customer service time, an opportunity constrained programming model with minimum total vehicle delivery cost is established. Compared with the standard genetic algorithm and particle swarm algorithm, the algorithm can effectively avoid the algorithm falling into the local optimum (Goksal et al., 2013) [12]. For a class of stochastic demand, vehicle routing problems (stochastic vehicle routing problem, SVRP) are constructed by combining the statistical knowledge. The hybrid particle swarm optimization algorithm is proposed by combining population search and trajectory search to reduce the chance by introducing a guided local search (Marinakis et al., 2013) [13]. A hybrid behavior ant colony algorithm is used to solve the multidepot routing problem. The simulation results show that the improved hybrid behavior ant colony algorithm is effective (Narasimha et al., 2013) [14]. The open vehicle routing problem is studied in which the vehicle does not need to return to the depot after serving the last customer point. The results show that the hybrid ant colony optimization algorithm is an effective method for solving the open vehicle routing problem (Marinakis and Marinaki, 2014) [15]. It is due to the current environmental requirements with multipopulation metaheuristics for solving vehicle routing problems (Osaba and Díaz, 2016) [16]. It defines a decision model using the proposed methodological Jenks optimization approach for possible optimal locations of various facilities which can be used by the transport infrastructure (Fraile et al., 2016) [17].

At present, most of the researches mainly construct the objective function model by considering all kinds of costs in the process of vehicle delivery. In order to avoid duplication carbon emissions costs, transportation costs, and time costs, we should make the best balance between overall costs. The research process and results of vehicle routing optimization under low carbon angle are limited. Therefore, we can use the model in the future research. The new algorithm can be improved reasonably and different intelligent algorithms can also be designed to solve the model in order to compare the 
convergence and effectiveness of different solutions. In the environment of promoting low carbon logistics, optimizing vehicle routing is an effective way to realize energy saving and emission reduction. It is of great practical significance to study and analyze the carbon emissions in logistics and transportation.

\section{Green Vehicle Routing Optimization Based on Carbon Emission}

Many recent green vehicle routing papers have presented mathematical models that are designed to minimize fuel consumption and the environmentally damaging carbon emissions in the routing decisions related to route choice, the load, and the speed of the vehicle. In view of the increasingly severe environmental problems, carbon emissions are calculated in the traditional route optimization of logistics distribution. The optimization model of distribution path considering the carbon emission and transportation costs is formed and the mixed integer programming model is solved by a hybrid algorithm (Turkensteen, 2015) [18]. The vehicle routing problem with time window constraints with carbon emissions is an extension of the classical vehicle routing problem. Considering the problem of logistics network optimization of carbon emissions, the model of distribution center selection and demand matching and the minimum carbon emission cost distribution model are constructed (Zhang et al., 2015) [19]. Multi-echelon distribution strategy is primarily to alleviate the environmental consequence of logistics operations. Simultaneously, carbon emission optimization targets and constraints have been found to be more complex. Finally, the important effects of carbon emissions and time windows on vehicle routing problem are analyzed (Li et al., 2016) [20]. With the development of economy and the deep understanding of the relationship between people and environment, the concept of green logistics will be more and more popular and recognized by ordinary consumers.

This illustration points out the need to specify the carbon emissions in the process of logistics and distribution which mainly come from the fuel consumption of transportation vehicles. The total carbon emission of the $E$ of different fuels has the following relation. The formula of the actually measured value $E$ is demonstrated as follows:

$$
\begin{aligned}
& E=\sum_{i=1}^{n} E_{i}=\sum_{i=1}^{n} f_{i} * \xi_{i} * F_{i} \\
& F_{i}=\left[u *\left(w_{0}+w\right)+\lambda * v^{2}\right] * d,
\end{aligned}
$$

where $E$ is the total carbon emission. $u$ is a constant relating to the condition of a road. $w_{0}$ is the empty weight of the transported vehicle. $w$ is the carrying capacity of the transport vehicle. $\lambda$ is the constant associated with the vehicle type of the vehicle. $v$ is the speed of the vehicle. $d$ is the distance traveled by the vehicle.

We also take the transportation route optimization question as the breakthrough point and analyze the green question of the logistics link. Consequently, we consider the problem of vehicle routing with time windows with carbon emission and speed optimization and we have also introduced a speed based carbon emission algorithm. The formula for total carbon emissions during vehicle route optimization is shown as follows:

$$
\begin{aligned}
\min & F(x, v, d, \alpha, \beta, \gamma) \\
= & \sum_{i=1}^{m} \sum_{j=1}^{n} \sum_{k=1}^{p}\left[\alpha\left(\frac{d_{i j}}{v_{i j}}+s_{i}\right)+(\beta h+\gamma) * \frac{E}{1000} * d_{i j}\right] \\
& * x_{i j k} \\
& \sum_{i=1}^{n} \sum_{j}^{m} x_{i j}=1 \\
& \sum_{j=1}^{m} \sum_{k=1}^{p} x_{j k}=1 \\
& \sum_{i=0}^{n} \sum_{j}^{m} x_{i j}=\sum_{j=0}^{m} \sum_{k=1}^{p} x_{j k} \leq P \\
& \sum_{i=1}^{n} q_{i} *\left(\sum_{i=0}^{n} x_{i j k}\right) \leq Q .
\end{aligned}
$$

The formula minimizes the total cost for the objective function. It includes three parts: vehicle cost, fuel consumption fee, and carbon emission cost. The total cost of the green path optimization objective function takes into account vehicle usage, fuel consumption, and carbon emission costs. The traditional path optimization problems that often take the total travel distance will also minimize the vehicle speed on the arcs as decision variables and the path optimization problem is the traditional rate as exogenous variables are usually constant. Fuel consumption and carbon emissions are closely related to speed. Taking speed as a decision variable will improve the global optimization effect of the model and will be more in line with the actual situation of traffic. In the current state of strongly advocated low carbon logistics environment, the study and analysis of carbon emissions in logistics and transportation have a very important practical significance. Optimization of vehicle routing is to realize the transportation of energy saving and emission reduction is an effective way as the basic reference model. We introduce the calculation method of speed based on carbon emissions and the arc velocity as decision variables. Considering vehicle routing problem, the carbon emissions green optimization model with time window is presented.

\section{Multiobjective Hybrid Quantum Immune Algorithm}

The proposed adaptive DE operator is able to improve both of the convergence speed and population diversity which are validated by the experimental studies. On the basis of immune multiobjective optimization, the distribution estimation algorithm (EDA) is introduced to model the evolutionary population. Based on this study, a hybrid optimization algorithm for complex multiobjective optimization problems is proposed (Lin et al., 2015) [21]. A new artificial 
immune algorithm is proposed to solve the multiobjective optimization problem. In the multiobjective immune algorithm using parallel mechanism, multiple subpopulations are established to perform immunization, respectively. The diversity of population can be effectively improved and the convergence can be accelerated (Shang et al., 2016) [22]. The new affinity allocation method effectively solves the selection difficulty problem. The algorithm can effectively remove the poor particles and save the better particles. Meanwhile, the evolutionary algorithm and the new update strategy are used to enhance the convergence speed and preserve the diversity of the algorithm (Liang et al., 2015) [23]. The ant colony optimization is used to solve the problem that there are compatibility constraints of different types of goods in the same vehicle. The efficiency of the ant colony optimization algorithm that is a technique for optimization which imitates the behavior of ants in search of food has been proved for vehicle routing problem in terms of the objective function (Sicilia et al., 2015) [24]. Because of its complexity, dynamics, and modeling difficulties, traditional methods have been unable to solve such optimization problems. The intelligent algorithm based on heuristic has certain advantages in solving such problems. In solving the multiobjective optimization problem, the artificial immune system provides new methods and ideas by referring to the principles and mechanisms of information processing in biological immune system.

The immune is the extraction of biological immune system features. A computational model is combined with engineering applications which mimics the biological immune process. The immune algorithm has good global searching ability and memory function. The antigen corresponds to the objective function of the optimization problem and the antibody corresponds to the solution of the optimization problem. The degree of approximation between the feasible solution and the optimal solution is described by the affinity of antigen and antibody. When the antigen is invaded, the system automatically produces the antibodies. The system is adaptive to the environment through the promotion and suppression reaction between antibodies. In the early stages of quantum immune colonial algorithm, the quantum probability distribution is relatively uniform. Moreover, the entropy of observed variables is higher. At this point, the effect of the current optimal individual is increased to accelerate the evolution process. In the latter part of evolution, the modulus of quantum probability is approaching to 0 and 1 .

In order to avoid the local minimum, the variance of random scatter of quantum population can be increased. If we can build a new model, we should consider both fuzziness and randomness and consider the relevance between the two practical applications. Consequently, the analysis and expression of uncertainty must be more scientific and comprehensive. To solve this problem, the academician $\mathrm{Li}$ Deyi proposed the idea of cloud model and subordinate language model. In the process of knowledge representation, we find out interesting things that the cloud model has the characteristics of uncertainty, stability, and change in uncertainty. It embodies the basic principle of evolution of natural species. We use normal cloud operator to complete the transformation from concept space to numerical space to

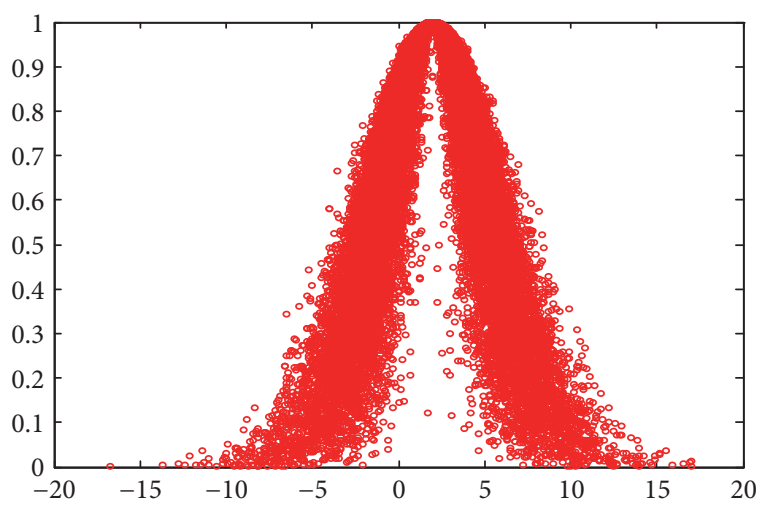

FIGURE 1: The simulation results of the cloud model operator.

realize immune operation. The simulation results of the cloud model operator are shown in Figure 1.

When the quantum antibody and the current optimal quantum antibody are in some specific states, the quantum bit in the quantum antibody does not ensure that the quantum bit of the current optimal quantum antibody is near. The resulting new quantum antibody may be far from the current optimal quantum antibody affecting the convergence of the algorithm. To improve the efficiency of the algorithm, the following quantum immune may be applied in the method. In view of the above problems, we use the cloud model to improve the quantum immune algorithm by the quantum rotation gate adjustment strategy. The implementation steps of the hybrid quantum immune algorithm are represented as follows.

Step 1 (the initial population is generated). $N$ antibodies are formed into initial groups and generate an initial quantum antibody population using the forward cloud.

Step 2 (selection operation). Select $N$ antibodies with the highest affinity from the population. The fitness of quantum antibodies in all populations was calculated and the fitness values of all antibodies were evaluated.

Step 3 (clonal amplification). The number of antibodies selected was expanded and the number was increased by cloning and amplification using a quantum rotating gate based on intercell chaotic ergodicity. According to the evaluation of fitness value, the excellent antibodies will enter into the next generation with greater probability.

Step 4 (mutation operations). Select $M$ antibodies with the lowest affinity from the population and mutate with a quantum revolving gate based on large interval chaotic traversal. According to the condition, the ill-adapted population is eliminated, and the seed antibody in the population is selected according to the fitness.

Step 5 (substitution operations). In the selection of antibodies for clonal expansion and mutation and the generation of new antibodies, the formula to replace antibodies with the 
lowest part of their affinity is used. Consequently, the population is qualitatively controlled according to the evolutionary situation.

Step 6 (optimal antibody retention). If the affinity of the best individual in the current generation group is lower than the previous generation, the best antibody in the previous generation will be used to replace the worst antibody of the current generation. Simultaneously, it can focus on the adaptive adjustment of rotation angle of quantum rotating gate. Accordingly, the quantum gates are applied to the probability amplitudes of all individuals in the population and the quantum antibody population is updated.

Step 7 (returns to Step 2, that is, loop calculation). Until the convergence condition or algebra is satisfied, the maximum limit is reached.

The algorithm can adaptively control the range of search space under the guidance of qualitative knowledge. The algorithm can avoid the local optimal solution in larger search space. The hybrid quantum immune algorithm has higher quality of solution. Moreover, the standard variance of the solution is small. Therefore, it shows that the performance of the algorithm is stable and robust.

\section{Example Simulation}

We use the cloud model operator instead of the rotating gate operator in the quantum immune algorithm to achieve the optimization function of the algorithm. Through the control of the parameters in the cloud model, the adaptive optimization mechanism of the algorithm is implemented. Concurrently, it can avoid local optimization effectively and it has a good advantage in convergence accuracy, search success rate, and iterative number. Considering the speed dependent time and time window constraints, the low carbon path arrangement will increase the total length of the distribution path. Nevertheless, it will not increase the economic burden of transportation enterprises and can significantly reduce the vehicle's carbon emissions and energy consumption. The uncertainty of customer demand time, the subjective preference decision of route optimization, and so on will affect the route optimization. These uncertainties also require dynamic real-time deployment and optimization as fuel consumption and carbon emissions are related. We interestingly discover that it can reduce carbon emissions which may save the total cost. Through the comparative study of various algorithm, the simulation results are shown in Figures 2-4.

Through a large number of numerical simulations, we discover that the hybrid multiobjective immune algorithm is a quick, effective, and satisfactory solution. The simulation results show that the hybrid algorithm can perform global search effectively. At the same time, the result of multiple experiments has testified and reduced the carbon emissions and the total cost by using the optimized speed path arrangement more than the fixed speed path arrangement.

There is a substitution relationship between carbon emission and route optimization time. There is a lot of carbon emission improvement space in the traditional vehicle routing. The experimental results of vehicle energy optimization and carbon emissions and speed are shown in Table 1 (vehicle cost (VC), fuel consumption (FC), emission carbon (EC), and total cost (TC)).

Through series of experiments, we consider that the current carbon trading market has a lower carbon price and the charging of carbon emissions will not significantly affect the enterprise's logistics and transportation arrangements and also cannot achieve the goal of effectively reducing carbon emissions. At last, we proved that the reducing carbon emissions will lead to an increase in route optimization time. The path planning strategy based on speed optimization can reduce the total cost and carbon emission more effectively than the fixed speed routing decision. The simulation results of the model with fixed speed and optimized speed are shown in Table 2.

By comparing the experimental and the simulation results, we found that the optimal speed can not only significantly reduce the total cost and travel time, but also can effectively reduce carbon while the speed is set to the total cost of the arc hour. Furthermore, it is showed that the path planning strategy based on speed optimization can reduce the total cost and carbon emissions more effectively than the fixed speed routing decision. The above data fully illustrate that when considering the shortest path, we should make full use of vehicles with larger carrying capacity for transportation. However, if the energy consumption problem is considered, the above analysis can show that the maximum energy consumption due to unit distance is too large and the shortest transportation path does not correspond to the minimum energy consumption. Therefore, considering time consuming, it should be integrated optimization.

The carbon emission intensity, energy efficiency, and energy structure of vehicle transportation are the forces to curb carbon emissions. The scale of production and the structure of production are the factors that stimulate carbon emissions. As the inhibition is limited, the gap between the contribution rate and the contribution rate is greater and the carbon emissions from vehicle transportation are increasing. The main reasons for the high carbon emissions are the expansion of the scale of transportation and the imbalance of production structure. The energy structure based on petroleum fossil energy is the root cause of high carbon emissions. In addition, energy efficiency of vehicle transportation is low which inhibits the carbon emission reduction rate. We must take full account of the energy consumption rate of various vehicles and fixed costs and other factors. The energy consumption of transport vehicles in the process and carbon emission factors were analyzed by LMDI method considering the energy emission intensity, energy structure, and energy intensity. The following experimental results of C-HQIA and the specifics are shown in Figures 5-7.

The experimental results show that the optimal arrangement of path velocity is more than the fixed speed. It can more effectively reduce the existing arrangements to replace the relationship between the total costs and carbon emissions of carbon emissions in the vehicle routing and travel time. We 

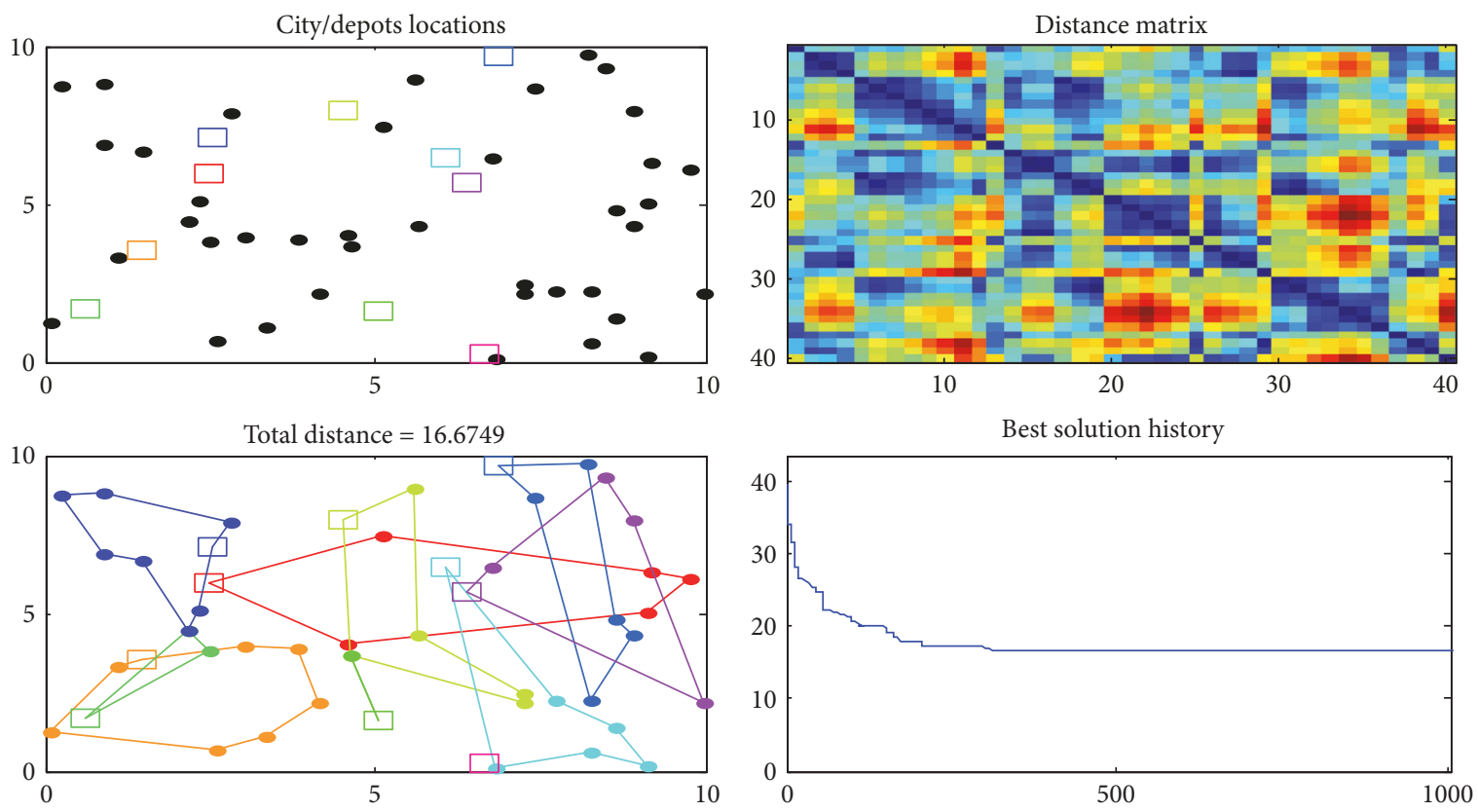

FIGURE 2: The simulation results of IA.
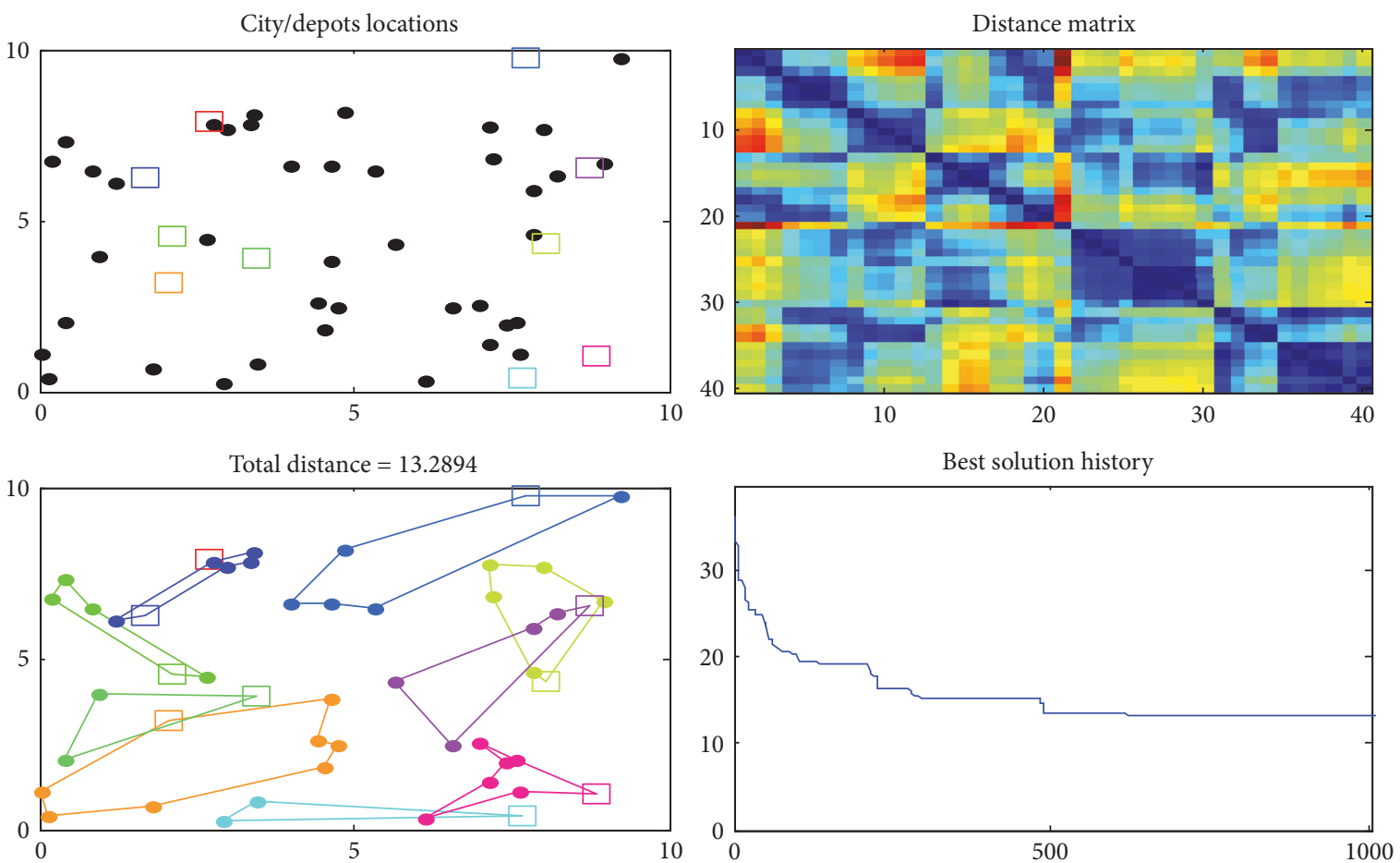

Figure 3: The simulation results of QIA.

find that reducing carbon emissions can lead to an increase in travel time. There is great carbon emission improvement space in traditional logistics transportation. As the carbon emissions of trucks are directly related to fuel consumption, reducing carbon emissions is conducive to saving the total transportation costs. Simultaneously, we discover that green path optimization is achieved mainly through the following three aspects. To begin with, we must make transportation scheduling optimization through optimization of line design and reasonable arrangements. Furthermore, it is to use multimodal transport to increase unit volume and improve the efficiency of green path optimization. Finally, it is reducing the total amount of carbon emissions to improve the green path optimization operation. 

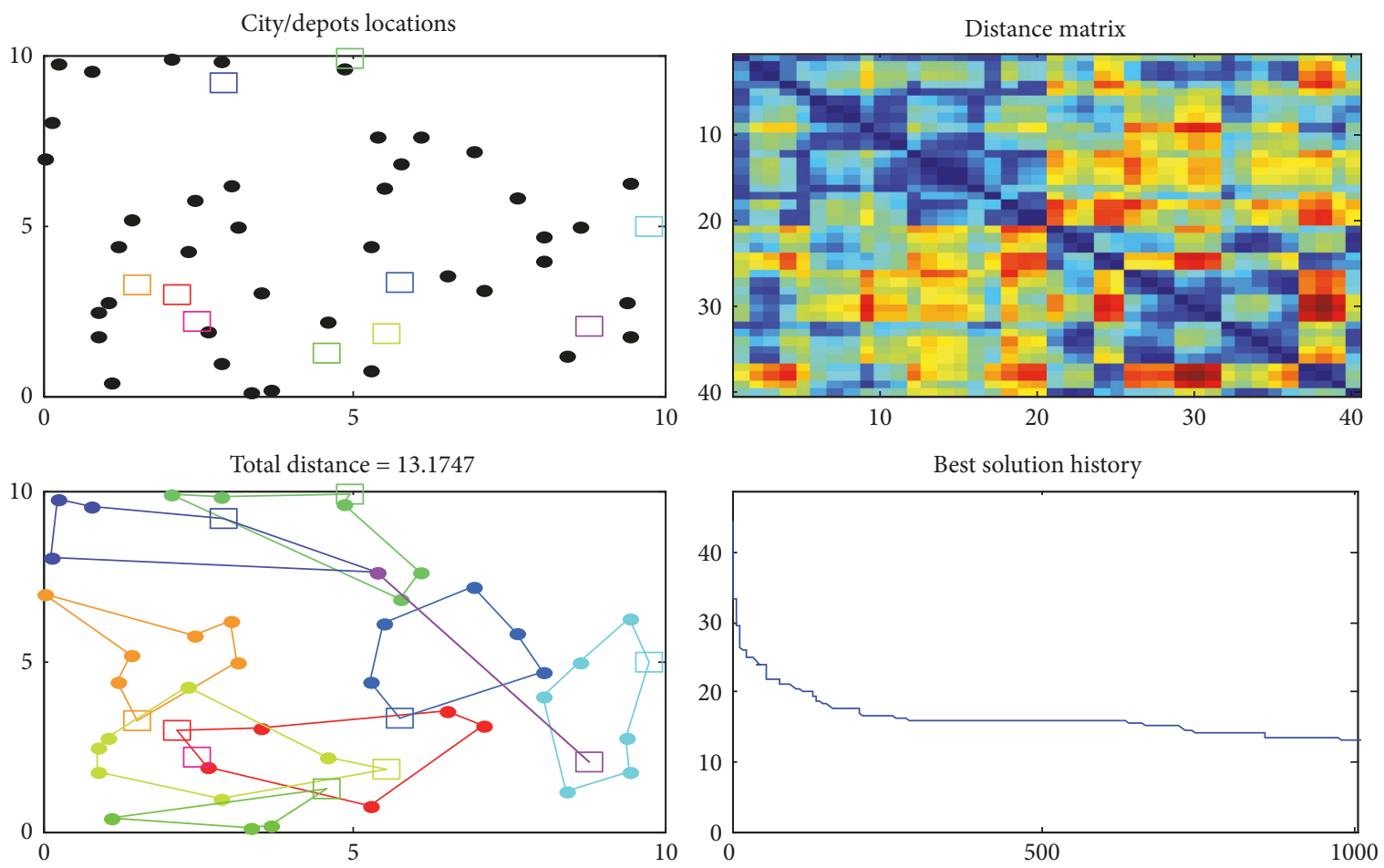

FIGURE 4: The simulation results of C-HQIA.

TABLE 1: The experimental results of vehicle energy optimization.

\begin{tabular}{lcccccccc}
\hline & $\mathrm{V}$ & $\mathrm{TT}$ & $\mathrm{TC}$ & $\mathrm{CO} 2$ & $\mathrm{VC} / \mathrm{TC}$ & $\mathrm{FC} / \mathrm{TC}$ & $\mathrm{EC} / \mathrm{TC}$ & \multicolumn{1}{c}{$\mathrm{S}$} \\
\hline RC201 & 79 & 49.52 & 8099.20 & 1325.53 & $51.43 \%$ & $45.98 \%$ & $0.87 \%$ & 101 \\
RC202 & 79 & 47.65 & 7240.45 & 1170.48 & $60.12 \%$ & $46.33 \%$ & $0.87 \%$ & 104 \\
RC101 & 75 & 57.05 & 7991.59 & 1112.04 & $56.48 \%$ & $37.01 \%$ & $0.71 \%$ & 159 \\
RC104 & 75 & 48.29 & 7025.05 & 1073.58 & $53.31 \%$ & $40.82 \%$ & $0.78 \%$ & 188 \\
R201 & 75 & 47.70 & 7032.03 & 998.18 & $57.79 \%$ & $39.97 \%$ & $0.75 \%$ & 73 \\
R202 & 79 & 46.25 & 6979.46 & 999.54 & $58.20 \%$ & $40.56 \%$ & $0.74 \%$ & 89 \\
R301 & 69 & 55.38 & 7902.15 & 999.83 & $62.54 \%$ & $34.90 \%$ & $0.73 \%$ & 179 \\
R301 & 76 & 46.02 & 6688.09 & 952.08 & $57.28 \%$ & $40.03 \%$ & $0.75 \%$ & 201 \\
C201 & 69 & 165.71 & 16680.10 & 653.02 & $87.76 \%$ & $10.18 \%$ & $0.20 \%$ & 139 \\
C202 & 83 & 163.05 & 16562.18 & 639.02 & $87.02 \%$ & $10.33 \%$ & $0.20 \%$ & 127 \\
C301 & 73 & 165.51 & 15730.21 & 472.21 & $90.29 \%$ & $8.07 \%$ & $0.17 \%$ & 199 \\
C302 & 72 & 160.78 & 15752.13 & 471.04 & $90.79 \%$ & $8.03 \%$ & $0.15 \%$ & 197 \\
Ave. & 75 & 87.74 & 10306.87 & 905.55 & $67.75 \%$ & $30.18 \%$ & $0.58 \%$ & 146.33 \\
\hline
\end{tabular}

\section{Conclusion}

Logistics is not only a major source of energy consumption, but also an important source of $\mathrm{CO} 2$ emissions. On the basis of analyzing the characteristics of vehicle routing problem with fuel consumption and $\mathrm{CO} 2$ emission factors, the corresponding optimization model is constructed and the algorithm based on hybrid quantum immune is proposed. According to the model and algorithm of numerical simulation, the experimental study shows that the shortest path route is not necessarily the minimum energy consumption path compared with traditional vehicle route. The shorter the path based on the vehicle routing $\mathrm{CO} 2$ emission and the total mileage, the lower the overall cost. The hybrid quantum immune algorithm is an effective solution algorithm to solve the vehicle routing problem with green. Hence, it can be characterized by its function of effectively processing the GVROP.

Considering the external cost of $\mathrm{CO} 2$ emissions during vehicle operation, the optimal vehicle path is inconsistent with the traditional vehicle path based on the shortest distance or time through a large number of experiments. In the vehicle routing results based on $\mathrm{CO} 2$ emissions, the optimal path is closely related to vehicle attributes, vehicle speed, and 
TABLE 2: The simulation results of the fixed speed and optimized speed.

\begin{tabular}{lcccccccc}
\hline & TT0 & TC0 & CO2 & $\sum$ TT & $\sum$ TC & $\sum$ CO2 & $\Delta$ TC & $\Delta E$ \\
\hline RC201 & 49.03 & 8097.23 & 1314.42 & 49.25 & 8080.02 & 1359.51 & $4.89 \%$ & $2.33 \%$ \\
RC202 & 44.67 & 7258.80 & 1152.21 & 44.33 & 7245.81 & 1145.62 & $7.56 \%$ & $1.78 \%$ \\
RC101 & 55.01 & 7999.08 & 1023.96 & 55.13 & 7989.93 & 1100.01 & $8.36 \%$ & $2.26 \%$ \\
RC104 & 45.20 & 6998.02 & 1056.46 & 45.20 & 6995.86 & 1032.23 & $5.33 \%$ & $14.34 \%$ \\
R201 & 47.05 & 7038.20 & 975.50 & 47.72 & 7033.24 & 979.21 & $5.75 \%$ & $2.54 \%$ \\
R202 & 46.21 & 6903.42 & 990.54 & 46.35 & 6885.42 & 946.88 & $5.36 \%$ & $3.37 \%$ \\
R301 & 57.20 & 7898.96 & 984.16 & 54.20 & 7895.81 & 984.27 & $5.24 \%$ & $2.21 \%$ \\
R301 & 44.02 & 6656.12 & 938.01 & 41.07 & 6644.12 & 932.12 & $4.39 \%$ & $4.34 \%$ \\
C201 & 165.40 & 16632.18 & 635.20 & 159.22 & 16575.21 & 630.89 & $2.34 \%$ & $2.03 \%$ \\
C202 & 163.59 & 16810.10 & 618.21 & 160.29 & 16467.23 & 614.32 & $2.11 \%$ & $2.07 \%$ \\
C301 & 156.01 & 15752.33 & 456.03 & 154.34 & 15691.42 & 442.89 & $2.09 \%$ \\
C302 & 160.02 & 15702.34 & 458.23 & 157.28 & 15689.55 & 454.56 & $2.07 \%$ \\
Ave. & 86.12 & 10312.23 & 883.58 & 84.53 & 10266.13 & 885.21 & $2.08 \%$ \\
\hline
\end{tabular}

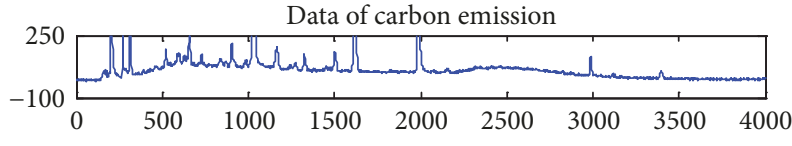

Baseline of carbon emission, as estimated by C-HQIA

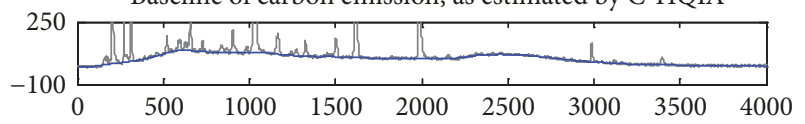

- Data of carbon emission

- Baseline of carbon emission
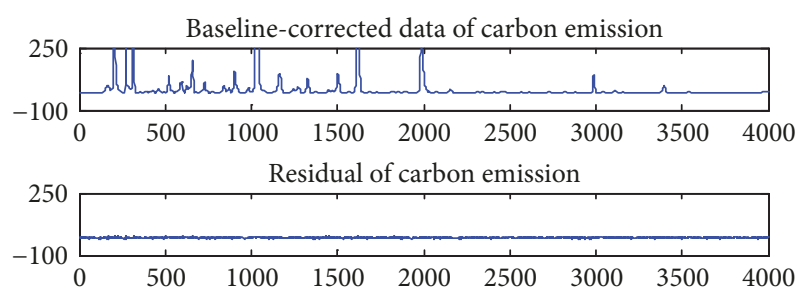

FIGURE 5: The simulation results considering the speed and energy.
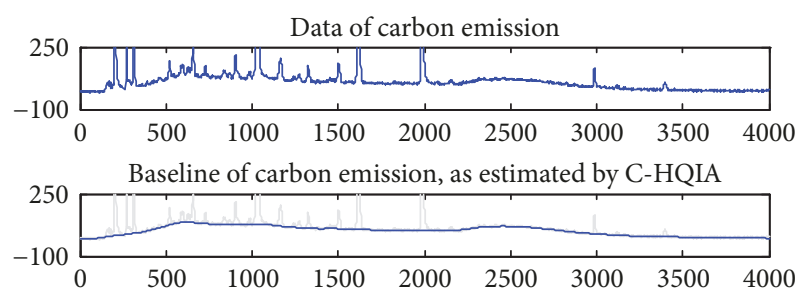

Data of carbon emission

— Baseline of carbon emission
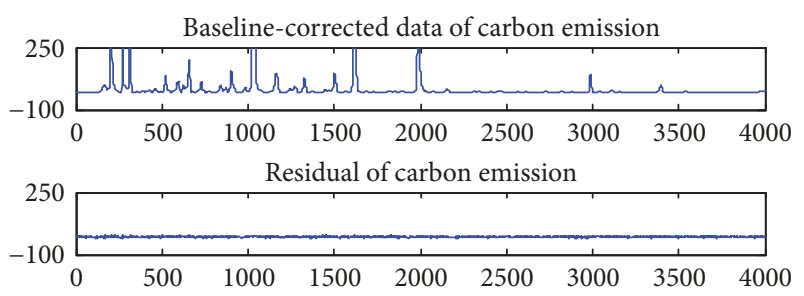

FIgURE 6: The simulation results considering the speed change.
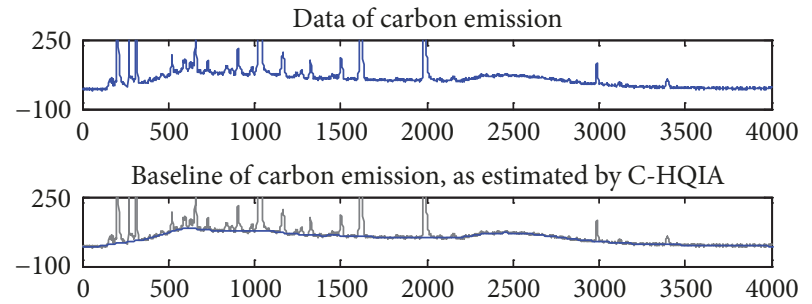

- Data of carbon emission

- Baseline of carbon emission
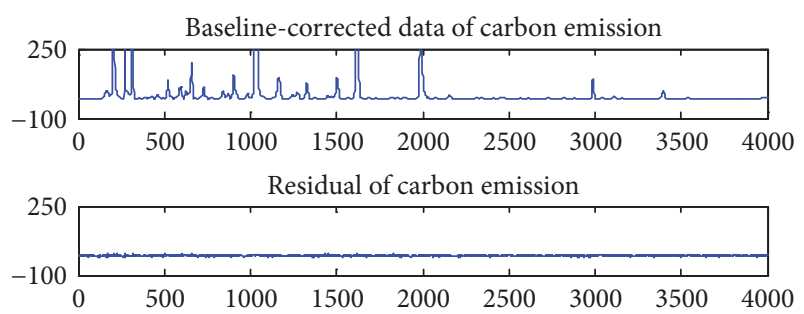

Figure 7: The simulation results considering the energy structure.

loading capacity. When the shortest path is considered, the vehicles with larger load capacity should be utilized as much as possible. As long as the fuel consumption is considered, the fuel consumption and the carbon emission cost are increased due to the excessive fuel consumption of the vehicle. As a result, distribution costs are lower with less loaded vehicles. Besides, vehicle $\mathrm{CO} 2$ emissions problems based on timevarying speed should also be considered to obtain the optimal time and optimal vehicle path. We also put forward that energy efficiency should be improved through the promotion of energy saving technology research. The negative effects of technological progress on China's carbon emissions are very significant. Improving energy efficiency has become an important means to promote energy conservation and emission reduction in China. Therefore, China should increase investment in advanced energy saving technologies while strengthening technical trade with developed countries. 


\section{Conflicts of Interest}

The authors declare that they have no conflicts of interest.

\section{Acknowledgments}

This research of work is partially supported by Hunan Provincial Natural Science Foundation (Grant no. 2017JJ2093), Hunan Provincial Department of Education Foundation (Grant no. 17C0667), the National Natural Science Foundation of China (Grant no. 70372039, Grant no. 70671037, and Grant no. 70971036), and the National Social Science Fund of China (Grant no. 13FGL005).

\section{References}

[1] A. M. F. M. AbdAllah, D. L. Essam, and R. A. Sarker, "On solving periodic re-optimization dynamic vehicle routing problems," Applied Soft Computing, vol. 55, no. 6, pp. 1-12, 2017.

[2] J. Qian and R. Eglese, "Fuel emissions optimization in vehicle routing problems with time-varying speeds," European Journal of Operational Research, vol. 248, no. 3, pp. 840-848, 2016.

[3] M. Marinaki and Y. Marinakis, "A glowworm swarm optimization algorithm for the vehicle routing problem with stochastic demands," Expert Systems with Applications, vol. 46, no. 3, pp. 145-163, 2016.

[4] W. Lefever, E.-H. Aghezzaf, and K. Hadj-Hamou, "A convex optimization approach for solving the single-vehicle cyclic inventory routing problem," Computers \& Operations Research, vol. 72, no. 8, pp. 97-106, 2016.

[5] Y. Zhao, L. Leng, Z. Qian, and W. Wang, "A discrete hybrid invasive weed optimization algorithm for the capacitated vehicle routing problem," Procedia Computer Science, vol. 91, no. 7, pp. 978-987, 2016.

[6] J. A. Sicilia, C. Quemada, B. Royo, and D. Escuín, "An optimization algorithm for solving the rich vehicle routing problem based on Variable Neighborhood Search and Tabu Search metaheuristics," Journal of Computational and Applied Mathematics, vol. 291, no. 2, Article ID 10105, pp. 468-477, 2016.

[7] F. Errico, G. Desaulniers, M. Gendreau, W. Rei, and L.-M. Rousseau, "A priori optimization with recourse for the vehicle routing problem with hard time windows and stochastic service times," European Journal of Operational Research, vol. 249, no. 1, pp. 55-66, 2016.

[8] N. Norouzi, M. Sadegh-Amalnick, and M. Alinaghiyan, "Evaluating of the particle swarm optimization in a periodic vehicle routing problem," Measurement, vol. 62, no. 2, pp. 162-169, 2015.

[9] L. J. Tan, F. Y. Lin, and H. Wang, "Adaptive comprehensive learning bacterial foraging optimization and its application on vehicle routing problem with time windows," Neurocomputing, vol. 151, no. 3, pp. 1208-1215, 2015.

[10] E. Jabir, V. V. Panicker, and R. Sridharan, "Multi-objective optimization model for a green vehicle routing problem," Procedia - Social and Behavioral Sciences, vol. 189, no. 3, pp. 3339, 2015.

[11] Q. Ding, X. Hu, L. Sun, and Y. Wang, "An improved ant colony optimization and its application to vehicle routing problem with time windows," Neurocomputing, vol. 98, no. 12, pp. 101-107, 2012.

[12] F. P. Goksal, I. Karaoglan, and F. Altiparmak, "A hybrid discrete particle swarm optimization for vehicle routing problem with simultaneous pickup and delivery," Computers \& Industrial Engineering, vol. 65, no. 1, pp. 39-53, 2013.

[13] Y. Marinakis, G.-R. Iordanidou, and M. Marinaki, "Particle Swarm Optimization for the vehicle routing problem with stochastic demands," Applied Soft Computing, vol. 13, no. 4, pp. 1693-1704, 2013.

[14] K. V. Narasimha, E. Kivelevitch, B. Sharma, and M. Kumar, "An ant colony optimization technique for solving min-max multi-depot vehicle routing problem," Swarm and Evolutionary Computation, vol. 13, pp. 63-73, 2013.

[15] Y. Marinakis and M. Marinaki, "A bumble bees mating optimization algorithm for the open vehicle routing problem," Swarm and Evolutionary Computation, vol. 15, pp. 80-94, 2014.

[16] E. Osaba and F. Díaz, "Design and implementation of a combinatorial optimization multi-population meta-heuristic for solving vehicle routing problems," International Journal of Interactive Multimedia and Artificial Intelligence, vol. 4, no. 2, pp. 89-90, 2016.

[17] A. Fraile, E. Larrodé, Á. Alberto Magreñán, and J. A. Sicilia, "Decision model for siting transport and logistic facilities in urban environments: A methodological approach," Journal of Computational and Applied Mathematics, vol. 291, no. 10, pp. 478-487, 2016.

[18] M. Turkensteen, "The accuracy of carbon emission and fuel consumption computations in green vehicle routing," European Journal of Operational Research, vol. 262, no. 2, pp. 647-659, 2017.

[19] J. Zhang, Y. Zhao, W. Xue, and J. Li, "Vehicle routing problem with fuel consumption and carbon emission," International Journal of Production Economics, vol. 170, pp. 234-242, 2015.

[20] H. Li, J. Yuan, T. Lv, and X. Chang, "The two-echelon timeconstrained vehicle routing problem in linehaul-delivery systems considering carbon dioxide emissions," Transportation Research Part D: Transport and Environment, vol. 49, no. 12, pp. 231-245, 2016.

[21] Q. Lin, Q. Zhu, P. Huang, J. Chen, Z. Ming, and J. Yu, "A novel hybrid multi-objective immune algorithm with adaptive differential evolution," Computers \& Operations Research, vol. 62, no. 10, pp. 95-111, 2015.

[22] R. Shang, B. Du, H. Ma, L. Jiao, Y. Xue, and R. Stolkin, "Immune clonal algorithm based on directed evolution for multi-objective capacitated arc routing problem," Applied Soft Computing, vol. 49, no. 12, pp. 748-758, 2016.

[23] Z. Liang, R. Song, Q. Lin et al., "A double-module immune algorithm for multi-objective optimization problems," Applied Soft Computing, vol. 35, no. 10, pp. 161-174, 2015.

[24] J. A. Sicilia, B. Royo, C. Quemada, M. J. Oliveros, and E. Larrodé, "A decision support system to long haul freight transportation by means of ant colony optimization," DYNA Ingeniería $e$ Industria, vol. 90, no. 1, pp. 105-113, 2015. 


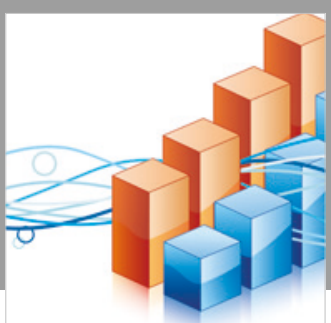

Advances in

Operations Research

\section{-n-m}
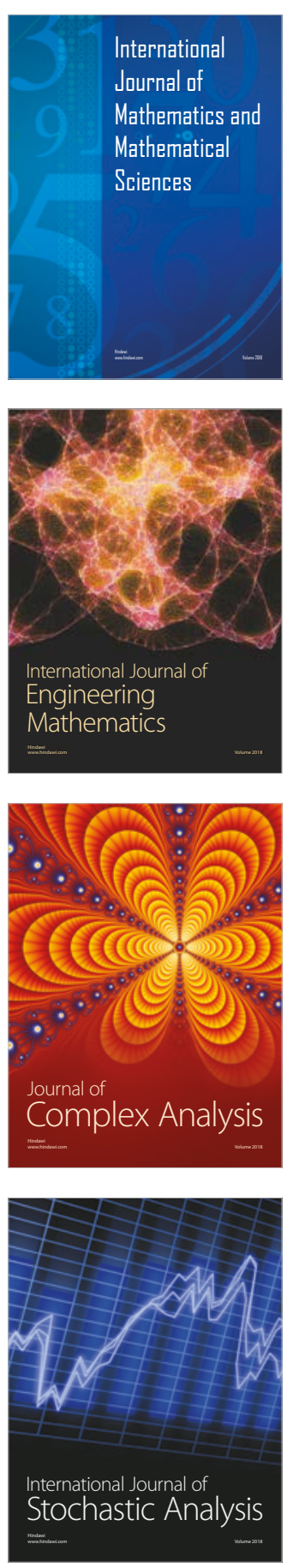
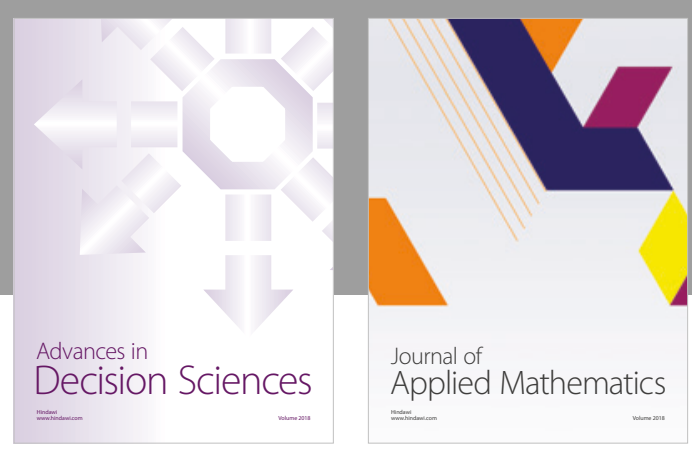

Journal of

Applied Mathematics
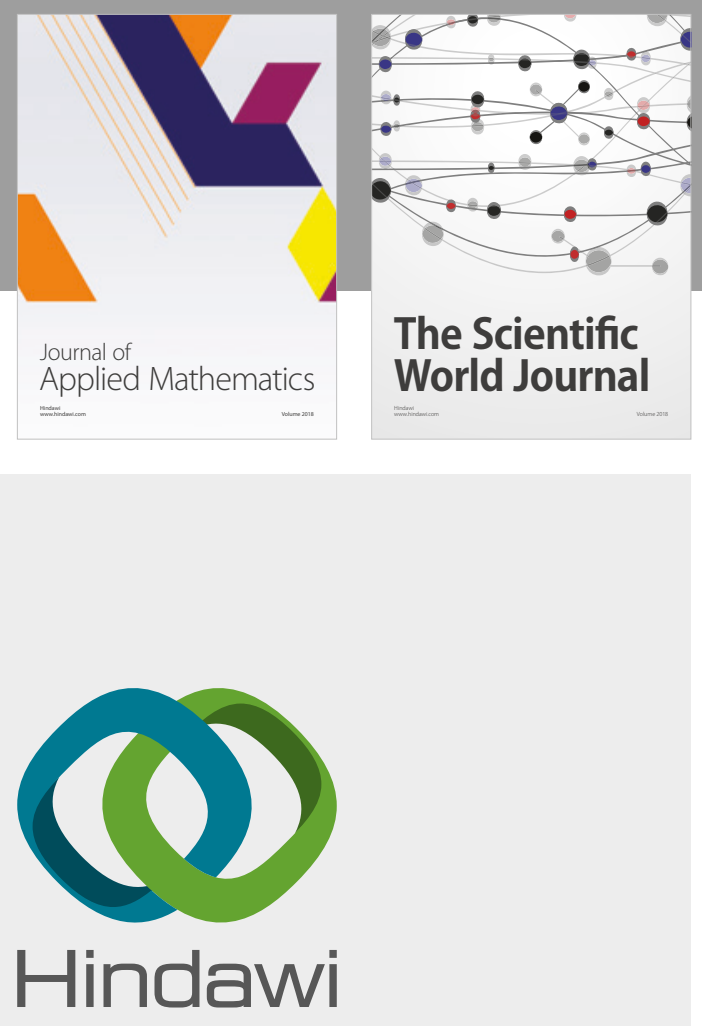

Submit your manuscripts at

www.hindawi.com

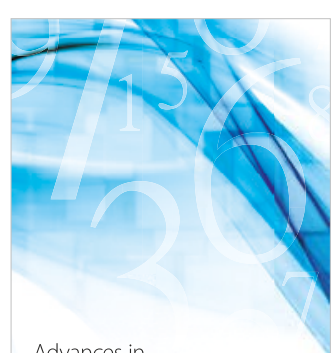

Advances in
Numerical Analysis
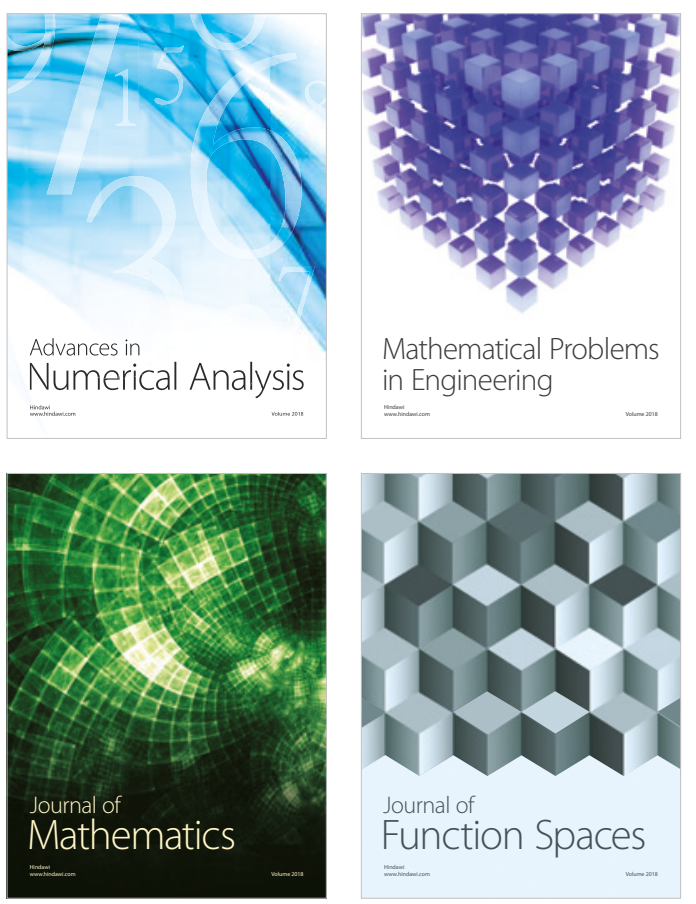

Mathematical Problems in Engineering

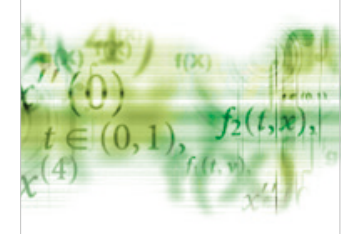

International Journal of

Differential Equations

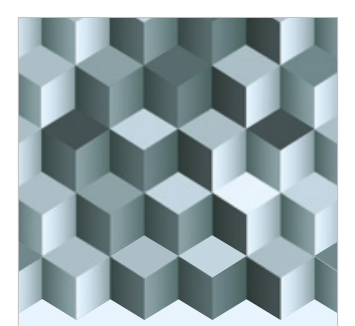

Journal of

Function Spaces

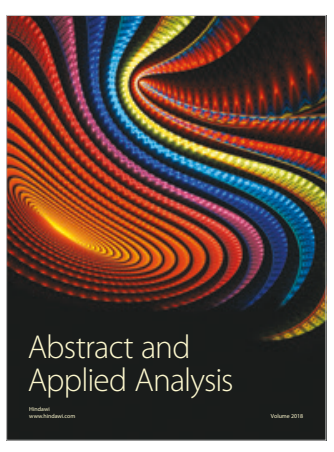

The Scientific

World Journal

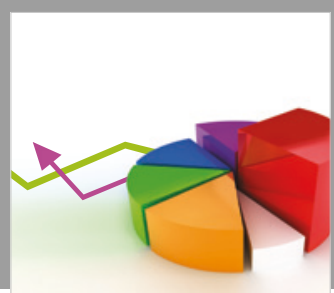

Journal of

Probability and Statistics
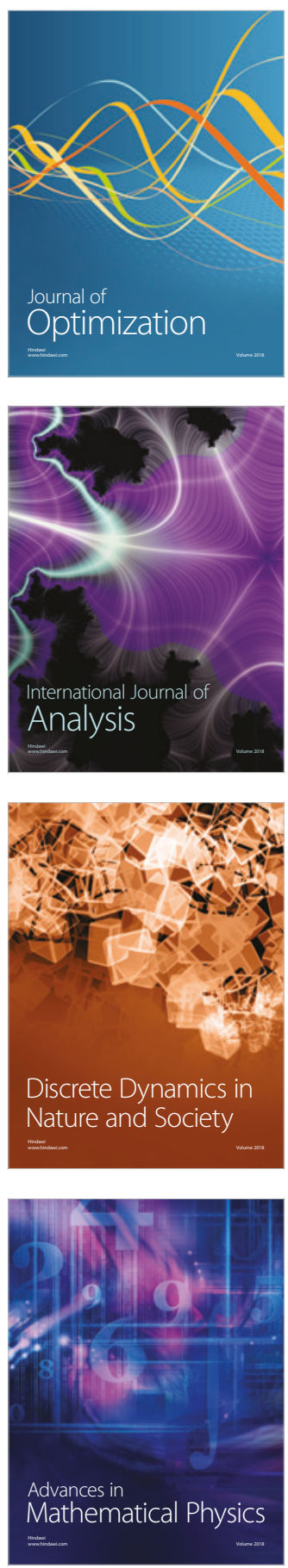